\title{
Pathological Findings of the Thyroid Tissue in a Patient with Euthyroid Graves' Disease
}

\author{
Nami Suzuki ${ }^{1}$, Ai Yoshihara ${ }^{1}$, Jaeduk Yoshimura Noh ${ }^{1}$, Ai Kozaki ${ }^{2}$, Toshu Inoue ${ }^{2}$, \\ Kiminori Sugino ${ }^{3}$ and Koichi Ito ${ }^{3}$
}

\begin{abstract}
Euthyroid Graves' disease (EGD) is a rare condition marked by the onset of thyroid-associated ophthalmopathy (TAO) in the absence of thyroid dysfunction. The pathogenesis of EGD remains unclear, and a consistent view of the pathological findings of the thyroid tissue has yet to be determined. We herein report a case of EGD in a 34-year-old woman with papillary carcinoma treated with total thyroidectomy. The entire thyroid specimen was investigated, and the thyroid tissue appeared normal. In this report, we discuss the relationship between the pathogenesis of TAO and thyroid dysfunction.
\end{abstract}

Key words: Euthyroid Graves' disease, thyroid-associated ophthalmopathy, pathological changes in thyroid tissue

(Intern Med 54: 2881-2884, 2015)

(DOI: 10.2169/internalmedicine.54.3346)

\section{Introduction}

Thyroid-associated ophthalmopathy (TAO) is characterized by immune-mediated inflammation of the extraocular muscles surrounding the orbital connective and adipose tissue and generally occurs in patients with hyperthyroidism due to Graves' disease. Euthyroid Graves' disease (EGD) is a condition marked by the appearance of TAO against a background of a normal thyroid function. Among the types of TAOs, EGD reportedly shows a prevalence of 0.7 $21 \%$ (1). Although EGD was first reported by Rundle and Wilson in 1945 (2), the pathogenesis continues to remain unclear; some authors have suggested that this pathology represents a prodromal stage of Hashimoto's thyroiditis or Graves' disease (3). Excluding results obtained from needle biopsies, a consistent view of the pathological findings of the thyroid tissue has yet to be determined, and case reports are lacking. We herein report a case of EGD with papillary carcinoma in which total thyroidectomy was performed.

\section{Case Report}

A 34-year-old Japanese woman presented to an ophthalmologist with a 5-month history of dry eyes and photophobia and a 3-month history of swelling of the right eyelid. After undergoing examinations, she was referred to our clinic for an evaluation of the thyroid function. She was euthyroid without treatment with any medications. The serum levels were as follows: free triiodothyronine, $2.8 \mathrm{pg} / \mathrm{mL}$ (reference range, $2.2-4.3 \mathrm{pg} / \mathrm{mL}$ ); free thyroxine, $1.44 \mathrm{ng} / \mathrm{dL}$ (reference range, 0.80-1.60 ng/dL); and thyrotropin, 0.97 $\mu \mathrm{U} / \mathrm{mL}$ (reference range, $0.20-4.50 \mu \mathrm{U} / \mathrm{mL}$ ). The titer of thyrotropin receptor antibodyies (TRAb) was $2.6 \mathrm{IU} / \mathrm{L}$, [thirdgeneration competitive receptor assay using thyroid stimulating hormone (TSH) receptor monoclonal antibody, electrochemiluminescence immunoassay; reference range, $<2.0 \mathrm{IU} /$ L] and the titer of thyroid-stimulating antibodies (TSAb) was $236 \%$ [Bioassay+Radio-immunoassay, (4); reference range, $<180 \%$ ], whereas negative results were obtained for thyroid-stimulating blocking antibodies (TSBAb) 34\%; [Bioassay+Radio-immunoassay, (5); reference range, <40\%], anti-thyroglobulin antibodies (TgAb) $11.1 \mathrm{IU} / \mathrm{mL}$; (enzyme-

${ }^{1}$ Department of Internal Medicine, Ito Hospital, Japan, ${ }^{2}$ Department of Ophthalmology, Olympia Eye Hospital, Japan and ${ }^{3}$ Department of Surgery, Ito Hospital, Japan

Received for publication May 27, 2014; Accepted for publication December 21, 2014

Correspondence to Dr. Jaeduk Yoshimura Noh, h-yoshimura@ito-hospital.jp 

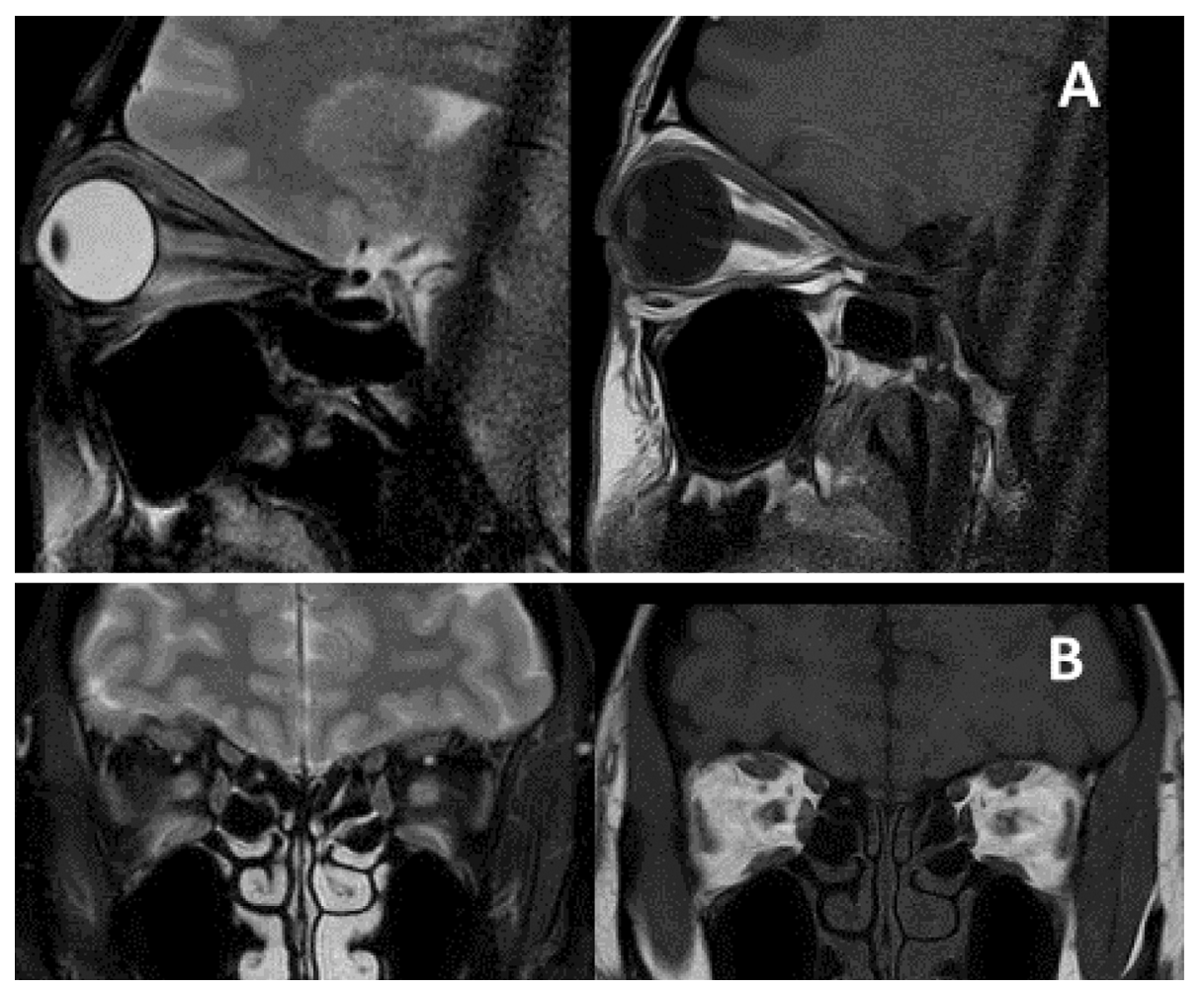

Figure 1. Magnetic resonance imaging of the orbit shows enlargement of the rectus and upper eyelid muscles.
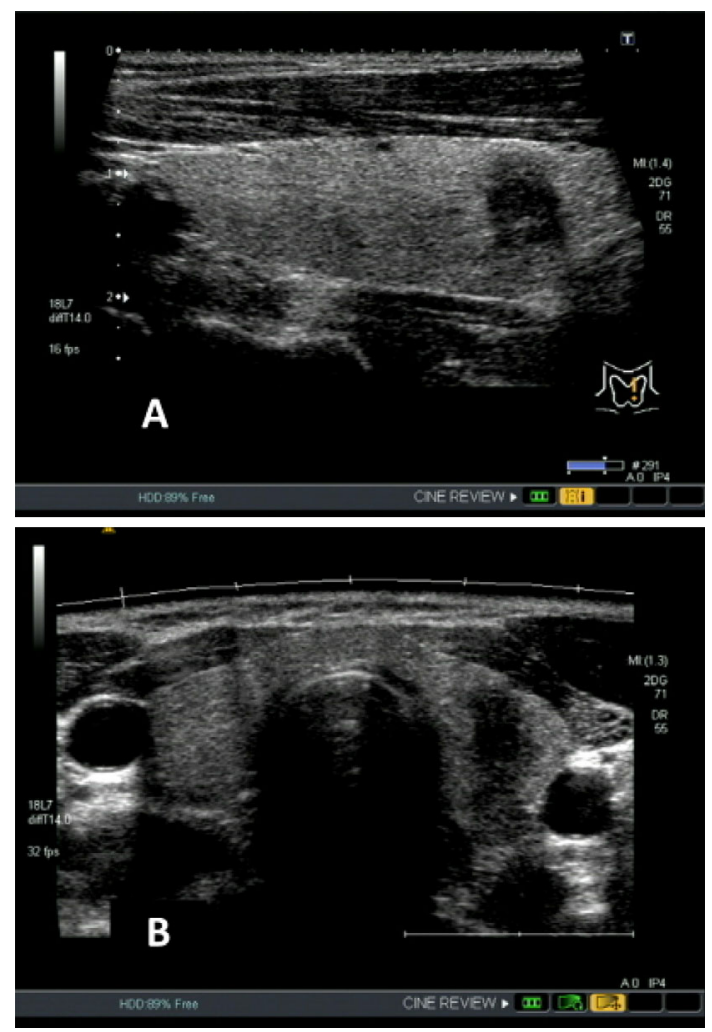

Figure 2. Thyroid imaging with ultrasonography. The thyroid appears isoechoic on ultrasound, which does not suggest the presence of Hashimoto's thyroiditis. 1) Longitudinal image: the tumor appears as a low-echoic lesion. 2) Axial image: the entire thyroid. immunoassay; reference range, $\leq 40 \mathrm{IU} / \mathrm{mL}$ ) and anti-thyroid peroxidase antibodies (TPOAb) $12.5 \mathrm{IU} / \mathrm{mL}$ (enzymeimmunoassay; reference range $\leq 28 \mathrm{IU} / \mathrm{mL}$ ). The thyroid was not palpable, and the estimated thyroid volume measured on ultrasonography was $12 \mathrm{~mL}$. Exophthalmos and retraction of the right eyelid were observed; the exophthalmometry measurements were $19 \mathrm{~mm}$ for the right eye and $17 \mathrm{~mm}$ for the left eye, with an eyelid fissure of $9 \mathrm{~mm}$ on the right and 8 $\mathrm{mm}$ on the left. Magnetic resonance imaging demonstrated hypertrophy of the right inferior rectus and upper eyelid muscles; however no tumors or inflammation were noted (Fig. 1). EGD was diagnosed based on these findings, and the Clinical Activity Score (CAS), which is used to evaluate the activity of TAO, was 1 . The local injection of triamcinolone into the right eyelid was performed to treat the ophthalmopathy. In addition, thyroid ultrasonography showed a 5-mm low-echoic nodule in the left lobe (Fig. 2), and an aspiration biopsy revealed papillary carcinoma. The positive TRAb and TSAb titers suggested a risk of hyperthyroidism in the near future. The patient was hoping to become pregnant and was worried about the potential side effects of antithyroid drugs that may be required during pregnancy. Considering these circumstances, total thyroidectomy was performed eight months after the diagnosis of EGD. During this period, the thyroid function remained within the normal limits, despite the positive TRAb and TSAb. A whole thyroid specimen was investigated; however, no findings typical of Hashimoto's thyroiditis, lymphocytic infiltration, fibrosis, Graves' disease or cellular hypertrophy or hyperplasia were seen, and the thyroid tissues appeared to be normal (Fig. 3). 


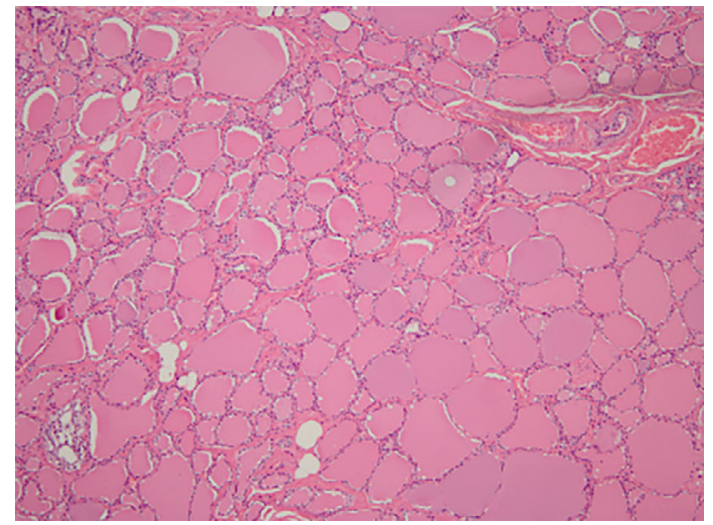

Figure 3. Hematoxylin and Eosin staining of the thyroid tissues showing neither hypertrophy nor hyperplasia. 100x magnification. No findings suggestive of Hashimoto's thyroiditis were identified.

Postoperatively, the patient has remained under treatment with oral thyroid hormone replacement therapy consisting of levothyroxine at a dose of $100 \mu \mathrm{g} /$ day. The TRAb titer became negative 7 months after thyroidectomy (1.1 IU/L).

\section{Discussion}

TAO is primarily related to hyperthyroidism, although it sometimes occurs with other types of thyroid disorders. Bartley et al. (6) reported that $90 \%$ of TAO patients have hyperthyroidism, $0.8 \%$ have hypothyroidism, $3.3 \%$ have Hashimoto's thyroiditis and $5.8 \%$ are euthyroid. The most common presenting symptoms of TAO are ocular surface discomfort, periorbital swelling, retro-orbital pain, gazeprovoked pain and diplopia (7). TAO is usually bilateral, with only $10-15 \%$ of patients showing asymmetrical symptoms (8).

Environmental factors, such as infection or smoking, have been suggested to influence the onset of TAO (9). A previous report showed that a male gender and advanced age at the time of diagnosis of TAO are related to severe ophthalmopathy (10). Our patient was a young woman who reported no history of severe infection or smoking.

Some hypotheses suggest that factors such as TRAb, insulin-like growth factor-1 receptor, cytokines and extraocular muscle antigens are involved in the development of TAO (11). Among these factors, only the TRAb titer was investigated in the present case.

There is also no consistent view as to the pathological findings of the thyroid tissue in patients with TAO. Some authors have suggested that this pathology represents a prodromal stage of Hashimoto's thyroiditis or Graves' disease (3), whereas others have reported that the histology of the thyroid tissue obtained via needle biopsy shows inflammatory and/or, degenerative changes or simple epithelial hyperplasia (12-14). Tamai et al. (3) reported the pathological findings of the thyroid tissue obtained via needle biopsy, with seven of nine cases exhibiting Hashimoto's thyroiditis, and the remaining displaying diffuse hyperplasia. Similarly, Kosugi et al. reported that two of their nine cases showed slight inflammatory or degenerative changes, while the remaining seemed to have normal thyroid tissue (14). Furthermore, Kasagi et al. (13) reported the histology in four cases. In that report, two patients positive for TSAb showed epithelial hyperplasia, while the other two patients demonstrated minimal degenerative changes. In addition, one of the latter patients was positive for anti-TgAb, anti-TPOAb, TBII and TSAb, whereas the other patient was positive only for TSAb. The discrepancies between the results of Tamai et al. and the findings observed in our case may be due to the fact that patients with Hashimoto's thyroiditis have tended to be collected in such reports; most previous cases have been positive for anti-thyroid antibodies. That is to say, the presence of a large goiter has a major effect on the decision to perform a biopsy.

In cases of Graves' disease, the thyroid tissue exhibits cellular hypertrophy and hyperplasia on microscopic examinations. In contrast, microscopic examinations in patients with Hashimoto's thyroiditis show many small follicles, a decreased amount of colloid particles and the extensive infiltration of lymphocytes, plasma cells and macrophages. In the current patient, no typical findings of Hashimoto's thyroiditis or Graves' disease were evident in a whole thyroid specimen. Several hypotheses as to why EGD patients may remain in a euthyroid state have been put forward, including a small thyroid $(12,15)$, destruction of the thyroid due to Hashimoto's thyroiditis $(3,12,14,16,17)$ or the weaker effect of TSAb derived from EGD patients in stimulating cAMP production than that required for the synthesis or release of thyroid hormones compared to the hyperthyroidism observed in patients with Graves' disease (12, 14-17). However, which of these mechanisms are applicable remains uncertain. We have encountered patients with hyperthyroid Graves' disease in whom the thyroid was almost normal in size and reported in the present case that the thyroid tissue from a patient with EGD appeared to be completely normal. Considering these observations, although there may be a correlation between the thyroid function and the size of the thyroid, abnormal reactivity of the thyroid and/or the signaling system involving cAMP, the first two hypotheses appear unlikely in this case. On the other hand, the last hypothesis remains a possibility, although the cAMP titer is measured on radio-immunoassays in vitro, and we have no evidence regarding how TSAb may differently stimulate cAMP production in vivo. Sato et al. reported a significant correlation between the TSAb titer and the thyroid hormone-releasing activity in an experiment using porcine thyrocytes (18); however, the results were obtained in vitro. In addition, as we were unable to examine the cAMP titer in the thyroid follicular cells from the current patient in vivo, it was not possible to clarify the relationship between ophthalmopathy and the thyroid function in this case. Further analyses are expected. 
The authors state that they have no Conflict of Interest (COI).

\section{Acknowledgement}

We are grateful to the pathologist and pathological technologists for their valuable assistance and recommendations.

\section{References}

1. Khoo DH, Eng PH, Ho SC, et al. Graves' ophthalmopathy in the absence of elevated free thyroxine and triiodothyronine levels: prevalence, natural history, and thyrotropin receptor antibody levels. Thyroid 10: 1093-1100, 2000.

2. Rundle FF, Wilson CW. Asymmetry of exophthalmos in orbital tumor and Graves's disease. Lancet 245: 51-52, 1945.

3. Tamai $\mathrm{H}$, Nakagawa T, Ohsako $\mathrm{N}$, et al. Changes in thyroid functions in patients with euthyroid Graves' disease. J Clin Endocrinol Metab 50: 108-112, 1980.

4. Yoshimura-Nor J. Clinical evaluation of thyroi stimulating antibody by yamasa TSAb kit. Horumon to Rinsho (Clinical Endocrinology) 42: 167-172, 1994 (in Japanese).

5. Kasagi K. Studies on the clinical application of TSBAB measurement using TSAb kit Yamasa. Horumon to Rinsho (Clinical Endocrinology) 46: 913-924, 1998 (in Japanese).

6. Bartley GB, Fatourechi V, Kadrmas EF, et al. Clinical features of Graves' ophthalmopathy in an incidence cohort. Am J Ophthalmol 121: 284-290, 1996.

7. Mohaseb K, Linder M, Rootman J, et al. Development and validation of a patient symptom questionnaire to facilitate early diagnosis of thyroid-associated orbitopathy in graves' disease. Orbit 27: 419-425, 2008

8. Eckstein AK, Losch C, Glowacka D, et al. Euthyroid and primarily hypothyroid patients develop milder and significantly more asymmetrical Graves ophthalmopathy. Br J Ophthalmol 93: 10521056, 2009.

9. Krassas GE, Wiersinga W. Smoking and autoimmune thyroid dis- ease: the plot thickens. Eur J Endocrinol 154: 777-780, 2006.

10. Manji N, Carr-Smith JD, Boelaert K, et al. Influences of age, gender, smoking, and family history on autoimmune thyroid disease phenotype. J Clin Endocrinol Metab 91: 4873-4880, 2006.

11. Smith TJ, Tsai CC, Shih MJ, et al. Unique attributes of orbital fibroblasts and global alterations in IGF-1 receptor signaling could explain thyroid-associated ophthalmopathy. Thyroid 18: 983-988, 2008.

12. Kasagi K, Hatabu H, Tokuda $Y$, et al. Studies on thyrotrophin receptor antibodies in patients with euthyroid Graves' disease. Clin Endocrinol (Oxf) 29: 357-366, 1988.

13. Kasagi K, Hidaka A, Misaki T, et al. Scintigraphic findings of the thyroid in euthyroid ophthalmic Graves' disease. J Nucl Med 35: 811-817, 1994.

14. Kosugi $\mathrm{S}$, Inoue $\mathrm{D}$, Sugawa $\mathrm{H}$, et al. Similarity and dissimilarity between clinical and laboratory findings, especially anti-thyrotropin receptor antibody in ophthalmic Graves' disease without persistent hyperthyroidism and hyperthyroid Graves' disease. Endocrinol Jpn 37: 343-354, 1990.

15. Kasagi K, Hatabu H, Tokuda $\mathrm{Y}$, et al. Comparison of thyroid stimulating activities measured by cyclic AMP production, those by radioiodine uptake in FRTL-5 cells and TSH-binding inhibitory activities in patients with hyperthyroid and euthyroid Graves' diseases. Acta Endocrinol (Copenh) 117: 365-372, 1988.

16. Solomon DH, Chopra IJ, Chopra U, Smith FJ. Identification of subgroups of euthyroid graves's ophthalmopathy. N Engl J Med 296: 181-186, 1977.

17. Teng CS, Smith BR, Clayton B, et al. Thyroid-stimulating immunoglobulins in ophthalmic Graves' disease. Clin Endocrinol (Oxf) 6: 207-211, 1977.

18. Sato K, Yamazaki K, Yamada E, et al. Immunoglobulins of untreated Graves' patients with or without thyrotropin receptor antibody (determined by porcine thyrocytes) universally elicit potent thyroid hormone-releasing activity in cultured human thyroid follicles. Thyroid 9: 979-988, 1999.

(C) 2015 The Japanese Society of Internal Medicine http://www.naika.or.jp/imonline/index.html 